\section{Systemic lupus erythematosus and extreme thrombocytosis without autosplenectomy}

\author{
Austin Anderson \\ Department of Internal Medicine, \\ University of Florida College of Medicine, \\ Gainesville, FL, USA
}

\begin{abstract}
Systemic Lupus Erythematosus (SLE) is an autoimmune disorder characterized by multiorgan system involvement. Hematologic manifestations are common in SLE and include thrombocytopenia in as many as 50\% of patients. Thrombocytosis is much less common in SLE, occurring in less than $5 \%$ of patients, and is typically reported in association with autosplenectomy. The following case report describes a 35-year-old female patient who presented for evaluation with extreme thrombocytosis of unclear etiology. The patient was diagnosed with reactive thrombocytosis due to active SLE for which treatment with corticosteroids and plaquenil was provided with subsequent decline in platelet counts. This report highlights the importance of considering SLE in the differential for thrombocytosis despite its classic association with thrombocytopenia.
\end{abstract}

\section{Introduction}

Systemic Lupus Erythematosus (SLE) is an autoimmune disease of unknown etiology. The pathogenesis of SLE is thought to be multifactorial. Genetic, hormonal, immunologic, and environmental factors have been associated with the development of SLE. ${ }^{1-4}$ The disease is best known for a wide array of clinical manifestations due to its multi-organ system involvement. Hematologic manifestations of SLE classically include anemia, leukopenia, and thrombocytopenia. Thrombocytopenia, in particular, has been found to occur in as many as $25-50 \%$ of patients with SLE. ${ }^{5}$

Thrombocytosis, defined as a platelet count greater than 400,000 cells $/ \mathrm{mm}^{3}$, is less common and has been found to occur in less than $5 \%$ of those with SLE. ${ }^{6}$ The etiology of thrombocytosis is distinguished between the presence (autonomous) or absence (reactive) of an underlying myeloproliferative disorder. Previous cases of thrombocytosis in patients with SLE have often been reported as reactive in nature due to autosplenectomy. The mechanism of thrombocytosis in the case of autosplenectomy is thought to involve silent splenic infarctions due to either recurrent vasculitis or arterial occlusion due to antiphospholipid syndrome. ${ }^{6,7}$ Cases of thrombocytosis in the setting of SLE are less often reported as reactive due to the underlying inflammatory state, and rarely reported in association with extreme thrombocytosis or a platelet count greater than $1,000,000$ cells $/ \mathrm{mm}^{3}$.

We report a case of extreme reactive thrombocytosis secondary to active SLE in the absence of autosplenectomy.

\section{Case Report}

A 35-year-old African American female with systemic lupus erythematosus was referred to our institution for evaluation of extreme thrombocytosis of unclear etiology. She was formally diagnosed in January 2015; however, her first manifestation of disease was three years earlier. She had a witnessed seizure in 2012 without offending agent or metabolic derangement. The next year, there was documentation that she had developed nasopharyngeal ulceration and non-erosive peripheral arthritis with bilateral hand involvement. She received her formal diagnosis in 2015 when she tested positive for anti-DNA antibody and ANA antibody. Medical and obstetric-gynecological histories were otherwise unremarkable. No previous treatment with steroids or steroid-sparing immunosuppressive therapy had been tried for management of her SLE.

The patient was referred to our institution for evaluation of extreme thrombocytosis of unclear etiology. She initially presented with a 1-week history of left foot pain. Physical exam was notable for cyanosis involving digits 2-5 of the left foot. The affected digits were cold and had diminished sensation to light touch. Dorsalis pedis and posterior tibial pulses were palpable. Computed tomography-angiogram of the left lower extremity was performed and revealed no evidence of acute arterial occlusion or areas of high-grade vascular stenosis. Heparin infusion was started for medical management of suspected microthrombi followed by conversion to coumadin and aspirin for continued therapeutic anticoagulation. Lab data revealed extreme thrombocytosis with a platelet count of 1.35 million.

The patient had no prior history of thrombocytosis. She denied infectious symptoms, history of splenectomy, abdominal trauma, recent surgical procedures, or acute blood loss. The patient reported no bleeding complications or vasomotor symptoms such as headache, visual change, light-headedness, or chest pain. Cytogenetic tests for essential thrombocytosis were negative for JAK-2 and calreticulin mutations. Bone marrow examination was per-
Correspondence: Austin Anderson, Department of Internal Medicine, University of Florida College of Medicine, 1600 SW Archer Rd, Gainesville, FL 32608, USA.

Tel.: + 1.352.413.1078.

E-mail: austinanderson2@gmail.com

Key words: Systemic lupus erythematosus; extreme thrombocytosis; reactive thrombocytosis.

Conflict of interest: the author declares no potential conflict of interest

Received for publication: 25 May 20115

Revision received: 13 July 2015.

Accepted for publication: 15 July 2015.

This work is licensed under a Creative Commons Attribution NonCommercial 3.0 License (CC BYNC 3.0).

(C) Copyright A. Anderson, 2015

Licensee PAGEPress, Italy

Rheumatology Reports 2015; 7:6035

doi:10.4081/rr.2015.6035

formed with biopsy revealing trilineage hematopoeisis and no evidence of megakaryocytic dysplasia. Abdominal ultrasound showed normal splenic size, architecture, and perfusion. Peripheral blood smear revealed no howell-jolly bodeies or target cells to suggest autosplenectomy or hyposplenism. Inflammatory markers were noted to be elevated (ESR 59 and CRP 6) with low C3 and C4 complement levels (65 and 9 respectively) suggestive of a reactive process driving the thrombocytosis in the setting of active SLE. Rheumatology was consulted advised treatment with high-dose prednisone $(40 \mathrm{mg})$ in addition to maintenance therapy with plaquenil. After 3-days of treatment, the thrombocytosis improved from 1.35 million to 750,000 per $\mathrm{mm}^{3}$.

\section{Discussion}

Extreme thrombocytosis is frequently the result of an underlying reactive process. It is less often due to an autonomous process such as myelodysplasia or myeloproliferation. One study of 280 patients with extreme thrombocytosis found that 82 percent had reactive thrombocytosis. The Further analysis of this subset of patients showed that infection was the most common culprit at 31 percent followed by hyposplenism at 19 percent. An underlying inflammatory state was responsible for just 9 percent of cases of reactive thrombocytosis. ${ }^{8}$ 
The patient in this report presented with a case of extreme thrombocytosis. Evaluation for autonomous thrombocytosis was performed with cytogenetic studies and a bone marrow examination. Mutations for JAK-2 and calreticulin were negative, and bone marrow biopsy revealed no signs of either myelodysplasia or myeloproliferation. Workup for reactive thrombocytosis was therefore pursued. The patient presented with no symptoms of signs to suggest underlying infection, and remained afebrile and without leukocytosis throughout the hospital course. As such, broad-spectrum infectious workup was not pursued; however, chest radiographs and urinalysis showed no signs of pneumonia or urinary tract infection respectively. Abdominal ultrasound was completed and found no radiographic evidence of asplenia or autosplenectomy. Peripheral blood smear was performed to evaluate for evidence of hyposplenism. No target cells or howell-jolly bodies were visualized. Evaluation for functional hyposplenism was limited in that peripheral blood smear examination has low sensitivity for the detection of mild functional hyposplenism. ${ }^{9}$ However, the detection of elevated inflammatory markers (ESR and CRP) and hypocomplementemia (C3 and C4) in the setting of active SLE, and reduction in platelet count following treatment with corticosteroids suggests an extreme thrombocytosis due to an underlying inflammatory state.

\section{Conclusions}

Although classically associated with thrombocytopenia, systemic lupus erythematosus is an under-reported and under-appreciated cause of thrombocytosis. Clinicians should consider active SLE in the evaluation for extreme thrombocytosis.

\section{References}

1. Schur PH. Genetics of systemic lupus erythematosus. Lupus 1995; 4:425.

2. Lahita RG. The role of sex hormones in systemic lupus erythematosus. Curr Opin Rheumatol 1999;11:352.

3. Theofilopoulos AN. The basis of autoimmunity. Part I. Mechanisms of aberrant self-recognition. Immunol Today
1995;16:90.

4. Cooper GS, Dooley MA, Treadwell EL, et al. Risk factors for development of systemic lupus erythematosus: allergies, infections, and family history. J Clin Epidemiol 2002;55:982.

5. Bhatt AS, Berliner N. Hematologic manifestations of SLE. In: Schur P, Massarotti E, eds. Lupus erythematosus: clinical evaluation and treatment. New York: Springer; 2012. pp 127-140.

6. Castellino G, Govoni M, Prandini N, et al. Thrombocytosis in systemic lupus erythematosus: a possible clue to autosplenectomy? J Rheumatol 2007;34:1497.

7. Abid N. Thrombocytosis in a patient with systemic lupus. J Pak Med Assoc 2013;63 :1305-6.

8. Buss DH, Cashell AW, O'Connor ML, et al. Occurrence, etiology, and clinical significance of extreme thrombocytosis: a study of 280 cases. Am J Med 1994;96:247.

9. Kirkineska L, Perifanis V, Vasiliadis T. Functional hyposplenism. Hippokratia 2014;18:7-11. 